\title{
THE BOUNDED FRAGMENT AND HYBRID LOGIC WITH POLYADIC MODALITIES
}

\author{
IAN HODKINSON
}

Imperial College London

\begin{abstract}
We show that the bounded fragment of first-order logic and the hybrid language with 'downarrow' and 'at' operators are equally expressive even with polyadic modalities, but that their 'positive' fragments are equally expressive only for unary modalities.
\end{abstract}

§1. Introduction. The bounded fragment of first-order logic is obtained by relativizing quantifiers to a relation symbol. It has been studied by, for example, Feferman \& Kreisel (1966); Feferman (1968); Areces et al. (2001); ten Cate (2005). It has the interpolation property, and a first-order formula is equivalent to a bounded formula iff it is invariant under generated submodels. The bounded fragment is also important in modal logic. In van Benthem (1985) it was shown that a first-order sentence in the signature of Kripke frames is preserved under disjoint unions, generated subframes, and bounded morphic images iff it is equivalent to a sentence of the form $\forall x \psi(x)$, where $\psi(x)$ is a 'positive' bounded formula. Goldblatt (1995) strengthened this by showing that for any class $\mathscr{K}$ of Kripke frames (for unary or polyadic modalities) that is closed under ultraproducts, the closure of $\mathscr{K}$ under the above three operations and ultraroots is defined by the set of all sentences of the above form $\forall x \psi(x)$ that are valid in $\mathscr{K}$. Since any elementary class is closed under ultraproducts, the modal logic of an elementary class of frames is always the modal logic of a class of frames defined by sentences very close to the bounded fragment.

Hybrid logic, an extension of modal logic, has been actively studied in recent years. For background, see, for example, ten Cate (2005) and Areces et al. (2001). As we will see below, in the latter paper it was shown that the bounded fragment and the hybrid language $\mathscr{H}(\downarrow, @)$ are equally expressive, by translating each fragment into the other. The translation of hybrid formulas to bounded ones works for modalities of any arity, but the converse translation was only given in the case where the modal operators are unary, and Hodkinson (2006, remark 3.16) stated that it does not appear to generalize to polyadic operators. Here we show that the translation does in fact generalize to polyadic operators, but that 'positive' bounded formulas are strictly more expressive than positive $\mathscr{H}(\downarrow$, @ $)$ formulas in the polyadic case.

In summary, the bounded fragment and $\mathscr{H}(\downarrow, @)$ are equally expressive for modalities of any arity. The 'positive' fragments of these languages are equally expressive only for unary modalities - this is a striking difference between the case of unary modalities, studied in the literature, and the polyadic case considered here.

Received: October 27, 2009

AMS 2000 subject classification: 03B45. 
§2. Definitions. Fix a modal signature with a set $\mathscr{P}$ of propositional atoms, a set $\mathscr{X}$ of state variables, and (for simplicity) a single modal diamond $\diamond$ and box $\square$, of arity $n$ for some arbitrary $n \geq 1$. (Nominals can be handled without difficulty, so for simplicity we omit them. Nullary modalities $(n=0)$ can also be handled.)

The hybrid language $\mathscr{H}(\downarrow, @)$ in this signature has syntax

$\alpha:=p|x| \top|\perp| \neg \alpha\left|\alpha_{1} \wedge \alpha_{2}\right| \alpha_{1} \vee \alpha_{2}\left|\diamond\left(\alpha_{1}, \ldots, \alpha_{n}\right)\right| \square\left(\alpha_{1}, \ldots, \alpha_{n}\right)\left|@_{x} \alpha\right| \downarrow_{x} \alpha$

for $p \in \mathscr{P}$ and $x \in \mathscr{X}$. We include $\vee, \square, \perp$ as primitive symbols as well as $\wedge, \neg, \diamond, \top$ because we do not want to treat them as abbreviations in the 'positive' fragments to be considered later. But $\rightarrow$ is an abbreviation: $\alpha \rightarrow \beta$ abbreviates $\neg \alpha \vee \beta$.

The corresponding bounded fragment of first-order logic is defined as follows. Let $R$ be an $(n+1)$-ary relation symbol. We will use the elements of $\mathscr{X}$ as first-order variables. For each $p \in \mathscr{P}$ we associate a unary relation symbol $P$, and write $\widehat{\mathscr{P}}$ for the set of these symbols. Then the atomic bounded formulas are $\mathrm{T}, \perp, P(x), R\left(x, y_{1}, \ldots, y_{n}\right)$, and $x=y$, for any $P \in \widehat{\mathscr{P}}$ and $x, y, y_{1}, \ldots, y_{n} \in \mathscr{X}$. If $\varphi, \psi$ are bounded formulas then so are $\varphi \wedge \psi, \varphi \vee \psi$, and $\neg \varphi$. Finally, if $\psi$ is a bounded formula, $x, y_{1}, \ldots, y_{n} \in \mathscr{X}$, and $x \notin$ $\left\{y_{1}, \ldots, y_{n}\right\}$, then $\exists y_{1} \ldots y_{n}\left(R\left(x, y_{1}, \ldots, y_{n}\right) \wedge \psi\right)$ and $\forall y_{1} \ldots y_{n}\left(R\left(x, y_{1}, \ldots, y_{n}\right) \rightarrow \psi\right)$ are also bounded formulas. The quantification here is 'bounded' by $R$. Again we will regard $\varphi \rightarrow \psi$ as an abbreviation of $\neg \varphi \vee \psi$, though here we could equally treat it as primitive.

Semantics for both languages is given by Kripke models $\mathscr{M}=(W, R, h)$, where $W \neq$ $\emptyset, R \subseteq W^{n+1}$, and $h: \mathscr{P} \rightarrow \wp(W)$. (We use ' $R$ ' for both the relation symbol and the corresponding relation.) We also need an assignment: a function $g: \mathscr{X} \rightarrow W$. The semantics $\mathscr{M}, g \models \varphi$ of the bounded fragment is as usual for first-order logic: in particular, $\mathscr{M}, g \models P(x)$ iff $g(x) \in h(p)$, for $p \in \mathscr{P}$. The main clauses for $\mathscr{H}(\downarrow, @)$ are

- $\mathscr{M}, g, w \models p$ iff $w \in h(p)$, for $p \in \mathscr{P}$,

- $\mathscr{M}, g, w \models x$ iff $w=g(x)$, for $x \in \mathscr{X}$,

- $\mathscr{M}, g, w \models \diamond\left(\alpha_{1}, \ldots, \alpha_{n}\right)$ iff there are $w_{1}, \ldots, w_{n} \in W$ with $R\left(w, w_{1}, \ldots, w_{n}\right)$ and $\mathscr{M}, g, w_{i} \models \alpha_{i}$ for each $i=1, \ldots, n$,

- $\mathscr{M}, g, w \models \square\left(\alpha_{1}, \ldots, \alpha_{n}\right)$ iff for all $w_{1}, \ldots, w_{n} \in W$ with $R\left(w, w_{1}, \ldots, w_{n}\right)$, we have $\mathscr{M}, g, w_{i} \models \alpha_{i}$ for some $i \in\{1, \ldots, n\}$,

- $\mathscr{M}, g, w \models @_{x} \alpha$ iff $\mathscr{M}, g, g(x) \models \alpha$,

- $\mathscr{M}, g, w \models \downarrow_{x} \alpha$ iff $\mathscr{M}, g^{\prime}, w \models \alpha$, where $g^{\prime}$ agrees with $g$ except perhaps on $x$ (we write ' $g^{\prime}={ }_{x} g$ ' for this), and $g^{\prime}(x)=w$.

As usual, $\square\left(\alpha_{1}, \ldots, \alpha_{n}\right)$ is equivalent to $\neg \diamond\left(\neg \alpha_{1}, \ldots, \neg \alpha_{n}\right)$.

§3. Known translations. Let us recall some existing translations from the literature. Areces et al. (2001, §3.1) define a 'standard first-order translation' of $\mathscr{H}(\downarrow$, @ )-formulas with only unary diamonds (i.e., $n=1$ ). The standard translation of each formula lies in the bounded fragment. Unfortunately, as pointed out in ten Cate \& Franceschet (2005, $\S 2)$, the translation is not quite correct. It tries to minimize the number of variables used, and this causes problems with $\downarrow$. $^{1}$ If no special effort is made to economize on variables, the translation becomes correct, and it extends easily to the polyadic case, as follows.

1 ten Cate \& Franceschet (2005) give a different 'standard translation' of $\mathscr{H}(\downarrow, @)$ into first-order logic, using the clauses $\left(\downarrow_{x} \varphi\right)^{i}=\exists x\left(x=v_{i} \wedge \varphi^{i}\right)$ and $\left(@_{x} \varphi\right)^{i}=\exists v_{i}\left(v_{i}=x \wedge \varphi^{i}\right)$. This translation is not into the bounded fragment. 
Let $v_{0}, v_{1}, \ldots$, be new variables not in $\mathscr{X}$, and define for each $\mathscr{H}(\downarrow, @)$-formula $\alpha$ and each $i \geq 0$ a first-order formula $\alpha^{i}$ as follows:

$$
\begin{aligned}
p^{i} & =P\left(v_{i}\right) \\
x^{i} & =x=v_{i} \\
\top^{i} & =\top \\
\perp^{i} & =\perp \\
(\neg \alpha)^{i} & =\neg \alpha^{i} \\
\left(\alpha_{1} * \alpha_{2}\right)^{i} & =\alpha_{1}^{i} * \alpha_{2}^{i}, \text { for } * \in\{\wedge, \vee\} \\
\diamond\left(\alpha_{1}, \ldots, \alpha_{n}\right)^{i} & =\exists v_{i+1} \ldots v_{i+n}\left(R\left(v_{i}, v_{i+1}, \ldots, v_{i+n}\right) \wedge \bigwedge_{1 \leq j \leq n} \alpha^{i+j}\right) \\
\square\left(\alpha_{1}, \ldots, \alpha_{n}\right)^{i} & =\forall v_{i+1} \ldots v_{i+n}\left(R\left(v_{i}, v_{i+1}, \ldots, v_{i+n}\right) \rightarrow \bigvee_{1 \leq j \leq n} \alpha^{i+j}\right) \\
\left(@_{x} \alpha\right)^{i} & =\alpha^{i}\left(x / v_{i}\right) \\
\left(\downarrow_{x} \alpha\right)^{i} & =\alpha^{i}\left(v_{i} / x\right) .
\end{aligned}
$$

Here, $\varphi(x / y)$ denotes the result of substituting $x$ for every free occurrence of $y$ in $\varphi$. This does not cause clashes because no quantifiers $Q x$ or $Q v_{i}$ appear in $\alpha^{i}$, for any $Q \in\{\forall, \exists\}$ and $x \in \mathscr{X}$. It can be checked that $\alpha^{i}$ is always a bounded formula. We can call $\alpha^{0}$ the standard translation of $\alpha$. It is easily seen that for any model $\mathscr{M}=(W, R, h)$ and assignment $g: \mathscr{X} \cup\left\{v_{0}, v_{1}, \ldots\right\} \rightarrow W$, we have $\mathscr{M}, g, g\left(v_{0}\right) \models \alpha$ iff $\mathscr{M}, g \models \alpha^{0}$ for every $\mathscr{H}(\downarrow, @)$-formula $\alpha$.

For $n=1$, a converse translation $(\varphi \mapsto H T(\varphi))$ of the bounded fragment into $\mathscr{H}(\downarrow, @)$ was given in Areces et al. $(2001, \S 3.1){ }^{2}$

$$
\begin{aligned}
H T(P(x)) & =@_{x} p \\
H T(R(x, y)) & =@_{x} \diamond y \\
H T(x=y) & =@_{x} y \\
H T(\top) & =\top \\
H T(\perp) & =\perp \\
H T(\neg \varphi) & =\neg H T(\varphi) \\
H T(\varphi * \psi) & =H T(\varphi) * H T(\psi), \text { for } * \in\{\wedge, \vee\} \\
H T(\exists y(R(x, y) \wedge \varphi)) & =@_{x} \diamond \downarrow_{y} H T(\varphi) \\
H T(\forall y(R(x, y) \rightarrow \varphi) & =@_{x} \square \downarrow_{y} H T(\varphi) .
\end{aligned}
$$

2 In Areces et al. (2001), $\perp, \vee, \forall$ were abbreviations. We have added explicit clauses for them, obtained by working through their definitions. 
For any model $\mathscr{M}$, assignment $g$, and world $w$ of $\mathscr{M}$, we have $\mathscr{M}, g \models \varphi$ iff $\mathscr{M}, g, w \models$ $H T(\varphi)$ for every bounded formula $\varphi$.

§4. New translations. We now generalize the translation $H T$ to arbitrary $n$. We define for each bounded formula $\varphi$ an $\mathscr{H}\left(\downarrow\right.$, @ )-formula $\varphi^{*}$ as follows.

1. $P(x)^{*}=@_{x} p$

2. $R\left(x, y_{1}, \ldots, y_{n}\right)^{*}=@_{x} \diamond\left(y_{1}, \ldots, y_{n}\right)$

3. $(x=y)^{*}=@_{x} y$

4. $\mathrm{T}^{*}=\mathrm{\top}, \perp^{*}=\perp,(\neg \varphi)^{*}=\neg \varphi^{*},(\varphi \wedge \psi)^{*}=\varphi^{*} \wedge \psi^{*}$, and $(\varphi \vee \psi)^{*}=\varphi^{*} \vee \psi^{*}$

5. We now consider the case $\varphi=\exists y_{1} \ldots y_{n}\left(R\left(x, y_{1}, \ldots, y_{n}\right) \wedge \psi\right)$. The translation is easier to define if $x, y_{1}, \ldots, y_{n}$ are distinct, and we can suppose so without loss of generality. (E.g., $\exists y(R(x, y, \ldots, y) \wedge \psi)$ is logically equivalent to the bounded formula $\exists y y_{2} \ldots y_{n}\left(R\left(x, y, y_{2}, \ldots, y_{n}\right) \wedge \psi \wedge \bigwedge_{i=2}^{n} y_{i}=y\right)$, where $y_{2}, \ldots, y_{n}$ are new distinct variables.) Assuming inductively that $\psi^{*}$ has been defined, we let

$$
\begin{aligned}
\varphi^{*} & =@_{x} \alpha_{0}, \text { where } \\
\alpha_{0} & =\diamond\left(\downarrow_{y_{1}} @_{x} \alpha_{1}, \top, \ldots, \top\right), \text { where } \\
\alpha_{1} & =\diamond\left(y_{1}, \downarrow_{y_{2}} @_{x} \alpha_{2}, \top, \ldots, \top\right), \text { where } \\
& \vdots \\
\alpha_{n-1} & =\diamond\left(y_{1}, \ldots, y_{n-1}, \downarrow_{y_{n}} @_{x} \alpha_{n}\right), \text { where } \\
\alpha_{n} & =\psi^{*} .
\end{aligned}
$$

6. Let $\varphi=\forall y_{1} \ldots y_{n}\left(R\left(x, y_{1}, \ldots, y_{n}\right) \rightarrow \psi\right)$. Again, although not essential, it helps to suppose that $x, y_{1}, \ldots, y_{n}$ are distinct. This can be done by, for example, replacing $\forall y(R(x, y, \ldots, y) \rightarrow \psi)$ by $\forall y y_{2} \ldots y_{n}\left(R\left(x, y, y_{2}, \ldots, y_{n}\right) \rightarrow \psi \vee\right.$ $\neg \bigwedge_{i=2}^{n} y_{i}=y$ ), where $y_{2}, \ldots, y_{n}$ are new variables. Assuming that $\psi^{*}$ has been defined, we put

$$
\begin{aligned}
\varphi^{*} & =@_{x} \beta_{0}, \text { where } \\
\beta_{0} & =\square\left(\downarrow_{y_{1}} @_{x} \beta_{1}, \perp, \ldots, \perp\right), \text { where } \\
\beta_{1} & =\square\left(\neg y_{1}, \downarrow_{y_{2}} @_{x} \beta_{2}, \perp, \ldots, \perp\right), \text { where } \\
& \vdots \\
\beta_{n-1} & =\square\left(\neg y_{1}, \ldots, \neg y_{n-1}, \downarrow_{y_{n}} @_{x} \beta_{n}\right), \text { where } \\
\beta_{n} & =\psi^{*} .
\end{aligned}
$$

We now show that this translation preserves meaning, so that indeed every bounded formula is expressible in $\mathscr{H}(\downarrow, @)$.

THEOREM 4.1. For every model $\mathscr{M}=(W, R, h)$, every assignment $g: \mathscr{X} \rightarrow W$, and every $w \in W$, we have $\mathscr{M}, g \models \varphi$ iff $\mathscr{M}, g, w \models \varphi^{*}$ for every bounded formula $\varphi$.

Proof. The proof is by induction on $\varphi$. First, if $\varphi$ is $P(x)$, we have $\mathscr{M}, g, w \models P(x)^{*}$ iff $\mathscr{M}, g, g(x) \models p$, iff $g(x) \in h(p)$, iff $\mathscr{M}, g \models \varphi$. Second, $\mathscr{M}, g, w \models R\left(x, y_{1}, \ldots, y_{n}\right)^{*}$ 
iff $\mathscr{M}, g, g(x) \models \diamond\left(y_{1}, \ldots, y_{n}\right)$, iff there are $b_{1}, \ldots, b_{n} \in W$ with $R\left(g(x), b_{1}, \ldots, b_{n}\right)$ and $\mathscr{M}, g, b_{i} \models y_{i}$ for each $i$. Since $\mathscr{M}, g, b_{i} \models y_{i}$ iff $b_{i}=g\left(y_{i}\right)$, this is iff $R\left(g(x), g\left(y_{1}\right), \ldots, g\left(y_{n}\right)\right)$ and so iff $\mathscr{M}, g \models R\left(x, y_{1}, \ldots, y_{n}\right)$ as required. Third, $\mathscr{M}, g, w \models(x=y)^{*}$ iff $\mathscr{M}, g, g(x) \models y$, iff $g(x)=g(y)$, iff $\mathscr{M}, g \models x=y$.

The boolean cases are straightforward. So let $\varphi$ be $\exists y_{1}, \ldots, y_{n}\left(R\left(x, y_{1}, \ldots, y_{n}\right) \wedge \psi\right)$, where $x, y_{1}, \ldots, y_{n}$ are distinct, and inductively assume the result for $\psi$. The easiest proof for $\varphi$ is 'by inspection'. In more detail, we claim that for each $d=0, \ldots, n$, we have $\mathscr{M}, g, w \models \varphi^{*}$ iff

$\dagger(d)$ : there exist $a_{i}^{j} \in W$ for $1 \leq i \leq n$ and $1 \leq j \leq d$, and assignments $g=g^{0}=y_{1}$ $g^{1}=y_{2} g^{2} \cdots=y_{d} g^{d}$, with

- $a_{i}^{i}=a_{i}^{j}=g^{j}\left(y_{i}\right)$ whenever $1 \leq i \leq j \leq d$,

- $R\left(g(x), a_{1}^{j}, \ldots, a_{n}^{j}\right)$ whenever $1 \leq j \leq d$,

- $\mathscr{M}, g^{j}, g(x) \models \alpha_{j}$ whenever $0 \leq j \leq d$.

To prove the claim, first observe that $\dagger(0)$ is equivalent to $\mathscr{M}, g^{0}, g(x) \models \alpha_{0}$, and so to $\mathscr{M}, g, w \models \varphi^{*}$. Suppose $1 \leq d \leq n$. We show that $\dagger(d-1)$ and $\dagger(d)$ are equivalent. Clearly, $\dagger(d)$ implies $\dagger(d-1)$. Assume $\dagger(d-1)$ : so $\mathscr{M}, g^{d-1}, g(x) \models \alpha_{d-1}$. By definition of $\alpha_{d-1}$, there exist $a_{1}^{d}, \ldots, a_{n}^{d} \in W$ with $R\left(g(x), a_{1}^{d}, \ldots, a_{n}^{d}\right), \mathscr{M}, g^{d-1}, a_{i}^{d} \models y_{i}$ for $1 \leq$ $i<d$, and $\mathscr{M}, g^{d-1}, a_{d}^{d} \models \downarrow_{y_{d}} @_{x} \alpha_{d}$, whence there is $g^{d}=y_{d} g^{d-1}$ with $g^{d}\left(y_{d}\right)=a_{d}^{d}$ and $\mathscr{M}, g^{d}, g^{d}(x) \models \alpha_{d}$. With $\dagger(d-1)$, this implies that $a_{i}^{i}=g^{d-1}\left(y_{i}\right)=a_{i}^{d}=g^{d}\left(y_{i}\right)$ for $1 \leq i<d$. Noting that $g^{d}(x)=g(x)$, we see that $\dagger(d)$ holds. The claim now follows by induction on $d$.

$\dagger(n)$ implies that $(\dagger)$ there are $a_{1}^{1}, a_{2}^{2}, \ldots, a_{n}^{n} \in W$ with $R\left(g(x), a_{1}^{1}, \ldots, a_{n}^{n}\right)$, and an assignment $g^{n}$ agreeing with $g$ except perhaps on $y_{1}, \ldots, y_{n}$, with $g^{n}\left(y_{i}\right)=a_{i}^{i}$ for each $i$, and $\mathscr{M}, g^{n}, g(x) \models \psi^{*}$. Also, it is easily seen that $(\dagger)$ implies $\dagger(n)$, since we may take $a_{i}^{j}=a_{i}^{i}$ for every $i, j$ and define the $g^{j}$ accordingly. Inductively, (†) holds iff there are $a_{1}^{1}, a_{2}^{2}, \ldots, a_{n}^{n}, g^{n}$ as above, with $\mathscr{M}, g^{n} \models \psi$. But this is exactly what it means to say that $\mathscr{M}, g \models \varphi$.

Since $\left(\forall y_{1} \ldots y_{n}\left(R\left(x, y_{1}, \ldots, y_{n}\right) \rightarrow \psi(\psi)\right)^{*}\right.$ is equivalent to $\left(\neg \exists y_{1} \ldots y_{n}\right.$ $\left.\left(R\left(x, y_{1}, \ldots, y_{n}\right) \wedge \neg \psi\right)\right)^{*}$, this case follows from the preceding one.

Our translation generalizes the existing one given in $\S 3$ because for $n=1, \varphi^{*}$ is the same as $H T(\varphi)$ if we omit the unnecessary but convenient $@_{x}$ in $\alpha_{n-1}$.

For a formula $\varphi$, let $|\varphi|$ denote the length of (i.e., number of symbols in) $\varphi$. We count variables and relation symbols as 1 , so that, for example, $|P(x)|=4,\left|R\left(x, y_{1}, \ldots, y_{n}\right)\right|=$ $2 n+4,|\top \rightarrow \perp|=|\neg \top \vee \perp|=4$, and $\left|\downarrow_{x} @_{x} p\right|=5$. It can be checked that

$$
\begin{aligned}
\left|\exists y_{1} \ldots \exists y_{n}\left(R\left(x, y_{1}, \ldots, y_{n}\right) \wedge \psi\right)\right| & =|\psi|+4 n+7, \\
\left|\left(\exists y_{1} \ldots y_{n}\left(R\left(x, y_{1}, \ldots, y_{n}\right) \wedge \psi\right)\right)^{*}\right| & =\left|\psi^{*}\right|+2 n^{2}+5 n+2 . \\
\left|\forall y_{1} \ldots \forall y_{n}\left(R\left(x, y_{1}, \ldots, y_{n}\right) \rightarrow \psi\right)\right| & =|\psi|+4 n+8, \\
\left|\left(\forall y_{1} \ldots \forall y_{n}\left(R\left(x, y_{1}, \ldots, y_{n}\right) \rightarrow \psi\right)\right)^{*}\right| & =\left|\psi^{*}\right|+\left(5 n^{2}+9 n+4\right) / 2 .
\end{aligned}
$$

An induction now shows that $\left|\varphi^{*}\right| \leq|\varphi|^{2}$ for every bounded formula $\varphi$, so the translation $\left(\varphi \mapsto \varphi^{*}\right)$ is quadratic. If $n$ is fixed in advance, then since also $\left|\varphi^{*}\right| \leq(n+1)|\varphi|$, it is linear. 
There is an alternative translation $\varphi \mapsto \varphi^{* *}$. Just to give the idea, for $n=2$ we put

$$
\left(\exists y_{1} y_{2}\left(R\left(x, y_{1}, y_{2}\right) \wedge \psi\right)^{* *}=@_{x} \diamond\left(\downarrow_{y_{1}} @_{x} \diamond\left(\top, \downarrow_{y_{2}}\left(\psi^{* *} \wedge @_{x} \diamond\left(y_{1}, y_{2}\right)\right)\right), \top\right) .\right.
$$

§5. 'Positive' formulas. Finally, we consider 'positive' formulas. On the bounded side, Goldblatt (1995) calls a first-order formula essentially atomic if it is built from atomic formulas using $\wedge, \vee$, and bounded quantification of the form $\exists y_{1} \ldots y_{n}\left(R\left(x, y_{1}, \ldots, y_{n}\right) \wedge\right.$ $\varphi)$ and $\forall y_{1} \ldots y_{n}\left(R\left(x, y_{1}, \ldots, y_{n}\right) \rightarrow \varphi\right)$, where $x \notin\left\{y_{1}, \ldots, y_{n}\right\}$. See the Introduction for more on these formulas: in particular (cf. van Benthem, 1985), a bounded formula is preserved by bounded morphisms iff it is equivalent to an essentially atomic formula. The essentially atomic formulas are in a sense the 'positive' bounded formulas, but beware: because $\rightarrow$ involves negation, $\forall y_{1} \ldots y_{n}\left(R\left(x, y_{1}, \ldots, y_{n}\right) \rightarrow \varphi\right)$ is not a positive firstorder formula.

On the hybrid side, $\mathscr{H}(\downarrow, @)^{\text {pos }}$ really is the positive fragment of $\mathscr{H}(\downarrow, @)$. It is defined to consist of all $\mathscr{H}(\downarrow, @)$-formulas not involving $\neg$.

Referring back to $\S 3$, we see that for any $n$, the standard translation of any $\mathscr{H}(\downarrow \text {, @ })^{\text {pos }}$ formula is essentially atomic, and that for $n=1$, the translation $H T$ from the bounded fragment to $\mathscr{H}(\downarrow, @)$ turns essentially atomic formulas into $\mathscr{H}(\downarrow, @)^{\text {pos }}$-formulas. So if $n=1$, the two kinds of formula are equally expressive.

However, Litak has observed that for $n \geq 2$, the translation(s) given in $\S 4$ do not take essentially atomic formulas into $\mathscr{H}(\downarrow \text {, @ })^{\text {pos }}$. For example, if $n=2$, we have

$$
\left(\forall y z(R(x, y, z) \rightarrow \psi)^{*}=@_{x} \square\left(\downarrow_{y} @_{x} \square\left(\neg y, \downarrow_{z} @_{x} \psi^{*}\right), \perp\right),\right.
$$

which is not positive. Litak asked if this is inevitable. We now show that it is, because when $n \geq 2$, there are essentially atomic formulas with no equivalent in $\mathscr{H}(\downarrow \text {, @ })^{\text {pos }}$.

For notational simplicity, let $n=2$. If $(W, R)$ is a frame and $a \in W$ then $(W,\{(b, c)$ : $R(a, b, c)\})$ is a directed graph. Essentially atomic formulas can easily express that this graph has only reflexive or symmetric edges. We will show that these properties cannot be expressedin $\mathscr{H}(\downarrow, @)^{\text {pos }}$.

To do this, from now on we restrict attention to pointed frames $A=(W, R, r)$ with a distinguished element $r \in W$, such that all triples in $R$ have $r$ as their first element. A pair $\left(a_{0}, a_{1}\right) \in W^{2}$ with $\left(r, a_{0}, a_{1}\right) \in R$ is called an edge of $A$. Given another frame $B=\left(W^{\prime}, R^{\prime}, r^{\prime}\right)$ like this, a nonempty set $\mathscr{S}$ of partial functions from $W$ to $W^{\prime}$ is said to be an $A$-to- $B$ system if

(S1) for all $f \in \mathscr{S}$ and $a \in W$, we have $a=r$ iff $a \in \operatorname{dom} f$ and $f(a)=r^{\prime}$,

(S2) for every edge $\left(a_{0}, a_{1}\right)$ of $A$ and every $f \in \mathscr{S}$, there is an edge $\left(b_{0}, b_{1}\right)$ of $B$ such that $f \cup\left\{\left(a_{i}, b_{i}\right)\right\} \in \mathscr{S}$ for each $i<2$,

(S3) similarly, exchanging $A$ and $B$.

This definition can be worked up into an Ehrenfeucht-Fraïssé game for $\mathscr{H}(\downarrow, @)^{\text {pos }}$, but we do not need such sophistication here. Note that in (S2) we do not require that $f \cup$ $\left\{\left(a_{0}, b_{0}\right),\left(a_{1}, b_{1}\right)\right\} \in \mathscr{S}$. (S2) implies that $\mathscr{S}$ is a set of partial homomorphisms.

EXAMPLE 5.1. Let $A=(W, R, r)$ and $B=\left(W^{\prime}, R^{\prime}, r^{\prime}\right)$ be frames such that $W$ is infinite, $R=\{(r, a, a): a \in W \backslash\{r\}\}, W^{\prime} \neq\left\{r^{\prime}\right\}$, and $\left(r^{\prime}, b, b\right) \in R^{\prime}$ for every $b \in W^{\prime} \backslash\left\{r^{\prime}\right\}$. Then the set $\mathscr{S}$ of all finite partial functions $f: W \rightarrow W^{\prime}$ satisfying (S1) is an A-to-B system. 
Proof. We check condition (S2). Let $f \in \mathscr{S}$. Take any edge of $A$ : it is of the form $(a, a)$. If $a \in \operatorname{dom} f$, then $(f(a), f(a))$ is an edge of $B$ and $f \cup\{(a, f(a))\}=f \in \mathscr{S}$, as required. If $a \notin \operatorname{dom} f$ then choose any $b \in W^{\prime} \backslash\left\{r^{\prime}\right\}$. Then $(b, b)$ is an edge of $B$, and $f \cup\{(a, b)\} \in \mathscr{S}$ as required.

For (S3), let $f \in \mathscr{S}$ and let $\left(b_{0}, b_{1}\right)$ be an edge of $B$. As $f$ is finite and $W$ infinite, there is some $a \in W \backslash \operatorname{dom} f$. Then $(a, a)$ is an edge of $A$ and $f \cup\left\{\left(a, b_{0}\right)\right\}, f \cup\left\{\left(a, b_{1}\right)\right\} \in \mathscr{S}$, as required.

Recall that a hybrid formula is pure if it has no subformulas in $\mathscr{P}$. To evaluate pure formulas, we only need frames $(W, R)$, not models $(W, R, h)$.

LemMA 5.2. Suppose that $\mathscr{S}$ is an A-to-B system. Then for any pure formula $\alpha$ of $\mathscr{H}(\downarrow, @)^{\text {pos }}$, the following holds: for any $f \in \mathscr{S}$, any assignment $g: \mathscr{X} \rightarrow \operatorname{dom} f$, and any $a \in \operatorname{dom} f$, if $A, g, a \models \alpha$ then $B, f \circ g, f(a) \models \alpha$.

Proof. By induction on $\alpha$. We consider the main cases. Let $f, g, a$ be given. If $\alpha$ is a variable $x \in \mathscr{X}$, then $A, g, a \models x$ iff $a=g(x)$, which implies $f(a)=f \circ g(x)$, so that $B, f \circ g, f(a) \models x$. If $\alpha=@_{x} \beta$ and $A, g, a \models \alpha$, then $A, g, g(x) \models \beta$. Note that $g(x) \in \operatorname{dom} f$. So inductively, $B, f \circ g, f(g(x)) \models \beta$, and so $B, f \circ g, f(a) \models \alpha$. If $\alpha$ is $\downarrow_{x} \beta$ and $A, g, a \models \alpha$, then $A, g^{\prime}, a \models \beta$, where $g^{\prime}={ }_{x} g$ and $g^{\prime}(x)=a \in \operatorname{dom} f$. Inductively, $B, f \circ g^{\prime}, f(a) \models \beta$. So by semantics, $B, f \circ g, f(a) \models \alpha$ since $f \circ g^{\prime}={ }_{x} f \circ g$ and $f \circ g^{\prime}(x)=f(a)$.

Suppose $\alpha$ is $\square\left(\beta_{0}, \beta_{1}\right)$, and that $A, g, a \models \alpha$. If $f(a) \neq r^{\prime}$ then trivially, $B, f \circ$ $g, f(a) \models \alpha$. So assume that $f(a)=r^{\prime}$ and so $a=r$. To show that $B, f \circ g, f(a) \models$ $\alpha$, take any edge $\left(b_{0}, b_{1}\right)$ of $B$. By (S3), there is an edge $\left(a_{0}, a_{1}\right)$ of $A$ such that $f_{i}=$ $f \cup\left\{\left(a_{i}, b_{i}\right)\right\} \in \mathscr{S}$ for each $i<2$. Because $A, g, r \models \square\left(\beta_{0}, \beta_{1}\right)$, there is $i<2$ with $A, g, a_{i} \models \beta_{i}$. Note that $g: \mathscr{X} \rightarrow \operatorname{dom} f_{i}$ and $a_{i} \in \operatorname{dom} f_{i}$. Inductively, $B, f_{i} \circ g, b_{i} \models \beta_{i}$ as well. Hence, $B, f_{i} \circ g, f(a) \models \alpha$. But $f_{i} \circ g=f \circ g$ because $\operatorname{rng} g \subseteq \operatorname{dom} f$. Therefore, $B, f \circ g, f(a) \models \alpha$, as required. The case of $\diamond$ is similar.

Now let $W=\mathbb{N}$ and $R=\{(0, i, i): i>0\}$. Let $W^{\prime}=\{0,1,2\}$ and $R^{\prime}=$ $\{(0,1,1),(0,2,2),(0,1,2)\}$. Let $A=(W, R, 0)$ and $B=\left(W^{\prime}, R^{\prime}, 0\right)$. Let $\mathscr{S}$ be the set of all finite partial maps from $W$ to $W^{\prime}$ satisfying (S1). By Example 5.1, $\mathscr{S}$ is an $A$-to- $B$ system.

Let $\varphi$ be the essentially atomic formula $\forall y z(R(x, y, z) \rightarrow y=z)$. Suppose for contradiction that some formula $\alpha$ of $\mathscr{H}(\downarrow, @)^{\text {pos }}$ is equivalent to $\varphi$, in the sense that for any model $\mathscr{M}$, any assignment $g$ into $\mathscr{M}$, and any $a$ in $\mathscr{M}$, we have $\mathscr{M}, g \models \varphi$ iff $\mathscr{M}, g, a \models \alpha$. We can assume without loss of generality that $\alpha$ is pure, so we can work with frames instead of models. Let $g: \mathscr{X} \rightarrow W$ be given by $g(v)=0$ for all $v \in \mathscr{X}$. Clearly, $A, g \models \varphi$, and so $A, g, 0 \models \alpha$. Take any $f \in \mathscr{S}$. Then $g: \mathscr{X} \rightarrow \operatorname{dom} f$ and $0 \in \operatorname{dom} f$, so by lemma 5.2, $B, f \circ g, 0 \models \alpha$. But plainly, $B, f \circ g \forall \models \varphi$, since $(0,1,2) \in R^{\prime}$. This is a contradiction.

The same example shows that symmetry, expressible by the essentially atomic formula $\forall y z(R(x, y, z) \rightarrow R(x, z, y))$, is not expressible in $\mathscr{H}(\downarrow, @)^{\text {pos }}$. We can adapt the examples to arbitrary $n \geq 2$ by defining $R\left(a, a_{1}, \ldots, a_{n}\right)$ to hold iff $a=a_{3}=\cdots=a_{n}=r$ and $\left(a_{1}, a_{2}\right)$ is an edge as above.

The following may be worth investigating.

Problems 5.3. On which frame classes (e.g., for $n=2$ ) are positive hybrid formulas as expressive as essentially atomic ones? 
What can we say about modal logics of classes of frames defined by positive hybrid formulas, for $n \geq 2$ ?

For each $n \geq 2$, find a 'simple' fragment of $\mathscr{H}(\downarrow, @)$ whose formulas are preserved under bounded morphisms and can express all essentially atomic formulas.

\$6. Acknowledgments. Many thanks to the editor Patrick Blackburn and the anonymous referee, to Tadeusz Litak for the suggestion to write this note and for valuable discussions, and to Dirk Pattinson for pointing out a mistake.

\section{BIBLIOGRAPHY}

Areces, C., Blackburn, P., \& Marx, M. (2001). Hybrid logics: Characterization, interpolation, and complexity. Journal of Symbolic Logic, 66, 977-1010.

Feferman, S. (1968). Persistent and invariant formulas for outer extensions. Compositio Mathematica, 20, 29-52.

Feferman, S., \& Kreisel, G. (1966). Persistent and invariant formulas relative to theories of higher order. Bulletin of the American Mathematical Society, 72, 480-485.

Goldblatt, R. (1995). Elementary generation and canonicity for varieties of boolean algebras with operators. Algebra Universalis, 34, 551-607.

Hodkinson, I. (2006). Hybrid formulas and elementarily generated modal logics. Notre Dame Journal of Formal Logic, 47, 443-478.

ten Cate, B. (2005). Model theory for extended modal languages. PhD Thesis, University of Amsterdam.

ten Cate, B., \& Franceschet, M. (2005). On the complexity of hybrid logics with binders. In Ong, L., editor, Proceedings of Computer Science Logic 2005, Volume 3634 of Lecture Notes in Computer Science, pp. 339-354. Berlin/Heidelberg, Germany: Springer Verlag. van Benthem, J. (1985). Modal Logic and Classical Logic. Naples, Italy: Bibliopolis.

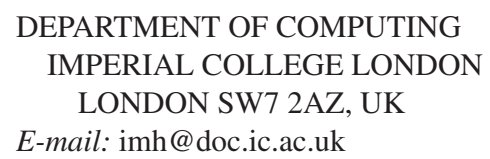

